\title{
Modal Gain Control in a Multimode Erbium Doped Fiber Amplifier Incorporating Ring Doping
}

\author{
Q. Kang, E.L. Lim, Y. Jung, J.K. Sahu, F. Poletti, S. Alam and D. J. Richardson \\ Optoelectronics Research Centre, University of Southampton, Southampton, SO17 1BJ, UK \\ *qk1g11@orc.soton.ac.uk
}

\begin{abstract}
We theoretically demonstrate the performance of a step index multimode (two mode-group) erbium-doped fiber amplifier with a localized erbium doped ring distribution for Space Division Multiplexed (SDM) transmission.
\end{abstract}

\section{Introduction}

With the information carrying capacity of conventional single-mode optical fiber close to fundamental limits in the laboratory there is increasing interest in the use of Space Division Multiplexing (SDM) as means to increase single fiber transmission capacity. Mode-DivisionMultiplexing (MDM) transmission over "fewmode fibers" (FMFs) represents one potential SDM approach and encouraging early results have been achieved. If such an approach is ever to be applied to long haul networks then the development of practical, high performance inline FMF optical amplifiers will be essential and initial results in this direction have also recently been reported ${ }^{1-3}$. Few mode erbium doped fiber amplifiers (FM-EDFAs) represent a particularly attractive approach, promising high-efficiency, high-gain devices with low differential modal gain (DMG) between all supported modes ${ }^{2,3}$. To date DMG in FM-EDFAs has been managed through control of the modal pump distribution ${ }^{2}$ (making for a complex and sensitive pump arrangement) with significant further improvements provided by simultaneous control of the fiber refractive-index profile (FRIP) and of the erbium ion distribution profile ${ }^{3}$. Whilst encouraging results have been demonstrated (e.g. gains of $>20 \mathrm{~dB}$ across the full C-band, with DMG values of $\angle 3 \mathrm{~dB}$ for all 6 modes in a two-mode fiber (TMF) supporting $L P_{01}$ and $L P_{11}$ modes $^{3}$ ) significant scope exists for new fiber concepts and designs providing simplified means of DMG control and improved overall gain performance.

In this paper we report the development and experimental validation of a modeling tool that allows the accurate prediction of FM-EDFA performance and numerically demonstrate a new fiber design concept that allows both for accurate DMG control whilst using a greatly simplified pumping arrangement using just the fundamental $\mathrm{LP}_{01}$ pump mode.

\section{Theoretical model}

Our FMF amplifier simulator is based on the rate and propagation equations dealing with multitransverse-spatial modes ${ }^{4,5}$. In this model, the following assumptions are made: (1) the EDFA is considered as a two-level system; (2) the erbium-doped fibers (EDFs) are weakly guided and the modes are well approximated by linearly polarized (LP) modes; (3) the fibers are uniform along the longitudinal direction and the mode coupling effects are negligible. The modal gain properties are determined by the interaction between spatially resolved gain in the active fiber and transverse mode decomposition of the optical field. Throughout this work, the emission/ absorption cross sections are derived from the literature ${ }^{6}$, where the fiber core composition $\left(\mathrm{Er}^{3+}\right.$ doped aluminosilicate fiber) is very similar to the EDF used in our experimental work ${ }^{3}$ and whose refractive index profile is shown in Fig. 1a (Fiber 1). The fluorescence spectrum of the Er-doped aluminosilicate glass has a peak at $\lambda_{\mathrm{s}}=1531 \mathrm{~nm}$ ( $7 \mathrm{~nm}$ wide) and a broader peak ( $20 \mathrm{~nm}$ wide) centered around $1545 \mathrm{~nm}$. For this reason, $1530 \mathrm{~nm}$ and $1550 \mathrm{~nm}$ are used as representative signal wavelengths for the two gain regions of interest. The key emission/absorption parameters used in the model are: $\sigma_{\mathrm{a}, 980 \mathrm{~nm}}=1.879 \times 10^{-25} \mathrm{~m}^{2}, \sigma_{\mathrm{e}, 980 \mathrm{~nm}}=0 \mathrm{~m}^{2}$, $\sigma_{\mathrm{a}, 1530 \mathrm{~nm}}=5.5 \times 10^{-25} \mathrm{~m}^{2}, \quad \sigma_{\mathrm{e}, 1530 \mathrm{~nm}}=5.674 \times 10^{-25} \mathrm{~m}^{2}$, $\sigma_{a, 1550 \mathrm{~nm}}=2.46 \times 10^{-25} \mathrm{~m}^{2}, \quad \sigma_{e, 1550 \mathrm{~nm}}=3.667 \times 10^{-25} \mathrm{~m}^{2}$. The $\mathrm{Er}^{3+}$ doping concentration is set to be $15 \times 10^{24} \mathrm{~m}^{-3}$ as estimated from the measured absorption $(10.1 \mathrm{~dB} / \mathrm{m})$ at the pump wavelength of $980 \mathrm{~nm}$. The background loss is neglected due to the short length of fibres required $(3-5 \mathrm{~m})$.

To illustrate use of the model we first show general results from modelling Fiber 1 which has a core diameter of $18 \mu \mathrm{m}$ and guides 3 spatial modes, $\mathrm{LP}_{01}, \mathrm{LP}_{11 \mathrm{a}}$ and $\mathrm{LP}_{11 \mathrm{~b}}$ within the $\mathrm{C}$-band. In the simulations, we use $L P_{11 a}$ to represent the $\mathrm{LP}_{11 \mathrm{~s}}$ mode group of the signal, and $\mathrm{LP}_{21 \mathrm{a}}$ to represent the $L P_{21 p}$ mode group of the pump. In Fig. $1 \mathrm{~b}$, we show the simulated DMG $\left(\mathrm{G}_{\mathrm{LP015}}{ }^{-}\right.$ $\left.\mathrm{G}_{\mathrm{LP11s}}\right)$ as a function of input signal power per mode for both Fiber 1 and a conventional Step Index (SI) TM-EDF (of equivalent effective NA and dopant concentration) when pumped by pure $L P_{01 p}$ and $L P_{21 p}$ modes with a fixed power of $250 \mathrm{~mW}$. The EDFAs were forward pumped and a fiber length of $3.5 \mathrm{~m}$ was used. As it shown 


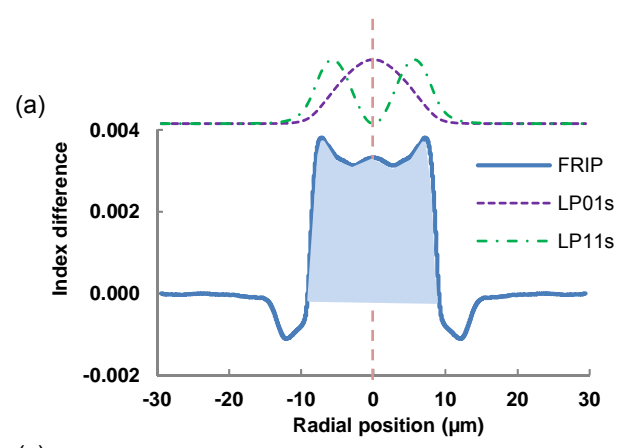

(c)

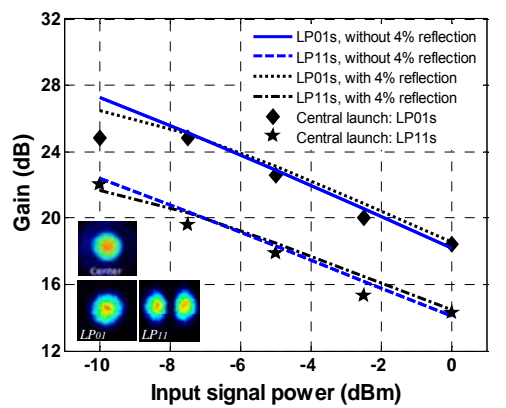

(b)

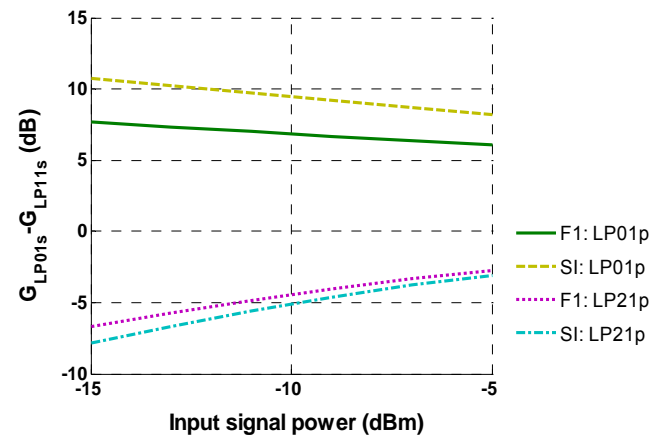

(d)

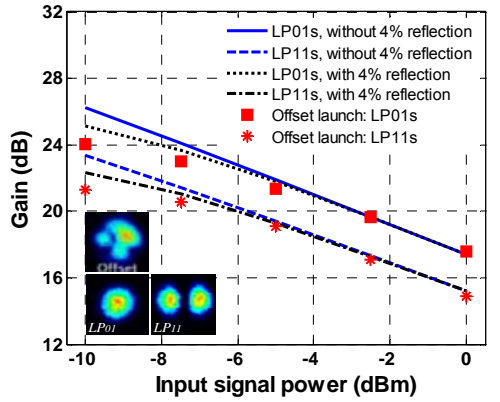

Fig. 1: The FRIP/dopant profile (shaded region) of Fiber 1 and supported signal modes. (b) DMG versus input signal power pumped by $\mathrm{LP}_{01 \mathrm{p}}$ and $\mathrm{LP}_{21 \mathrm{p}}$ for both Fiber 1 (shown as "F1") and step index EDF (shown as "SI"). Gain for $\mathrm{LP}_{01}$ and $\mathrm{LP}_{11}$ a modes versus input signal power under (c) central pump launch condition and (d) offset launch condition. The points represent experimental measurements; the lines show theoretical predictions.

in Fig. 1b, the gain differences between the two signal modes of Fiber 1 are significantly less than those of the step index TM-EDF, which validates that the EDF with a "batman" profile improves the amplifier performance in terms of reducing DMG. However, the simulations also indicate that a complex pump field distribution (i.e. a combination of $L^{2} P_{01 p}$ and $L P_{21 p}$ pump modes) is required for both fiber designs to minimise the DMG.

\section{Fitting the experimental data}

To validate the model predictions above we did detailed two mode group gain measurements using the experimental setup previously reported $^{3}$ and tried to fit these with our model. The fiber length was set to $3.5 \mathrm{~m}$ and counterpropogating pump condition were used. The wavelengths of both the signal modes were set to $1550 \mathrm{~nm}$ while the average input signal power per mode was changed from $-10 \mathrm{dBm}$ to $0 \mathrm{dBm}$. Results for both central and offset pump launch conditions $^{3}$, were considered in the modelling. As shown in Fig. 1c,d, the DMG exhibits a significant dependence on the pump modal configuration. For the central launch condition outlined in Fig. 1c, it was found that the best agreement between theory and experiment was achieved by assuming that $65 \%$ of the $280 \mathrm{~mW}$ total pump was split into $L_{01 p}$ mode with the remaining $35 \%$ in the $\mathrm{LP}_{21 p}$ mode. For offset pump launch conditions the best fit was achieved by assuming $40 \%$ of the pump in $\mathrm{LP}_{01 \mathrm{p}}$ and $60 \%$ in the $\mathrm{LP}_{21 \mathrm{p}}$, as shown in Fig. $1 \mathrm{~d}$. Although the experimental data points agree very well with the simulations at high input signal powers, a significant ( $3 \mathrm{~dB})$ discrepancy appears to occur at low powers until account is taken of the $4 \%$ Fresnel reflection from the flat input end face that is currently required in order to avoid significant mode-mixing at the signal launch. With this included, the apparent discrepancy at low powers is reduced from $3 \mathrm{~dB}$ to $1.5 \mathrm{~dB}$ and the general performance of the amplifier is well described over the full operating range. We thus conclude the scalar mode approximation and the various other assumption we have listed hold well in modeling our FMF-EDFA and can be reliably used to investigate other amplifier concepts and designs.

\section{Modal gain control in TM-EDFA using ring doping}

The physical origin of the DMG results from differences in the overlap of the pump, signal modes and the rare earth dopants. As first illustrated in Fig. 1a, we can substantially change the DMG by varying the Refractive Index $(\mathrm{RI})$ and rare-earth doping profiles within the fiber. In the case of F1 the dopant profile follows the RI profile of the fiber due to the specific fabrication process adopted but that need not be the case. For example, it is possible to envisage incorporating the dopant in an annular ring surrounding an undoped central core region of a step index fiber and which, as is clear from examination of Fig. 2a (Fiber 2), provides great scope for engineering the relative overlap of the $\mathrm{LP}_{01}$ and $L \mathrm{P}_{11}$ signal modes with the Er ions. 


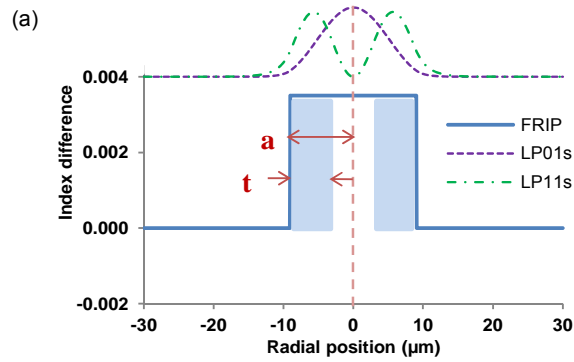

(b)

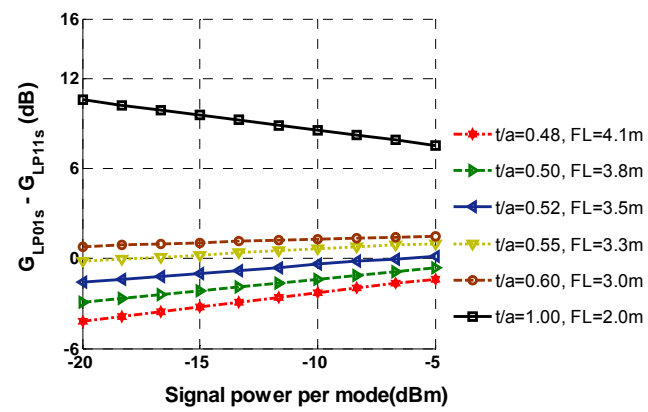

(c)

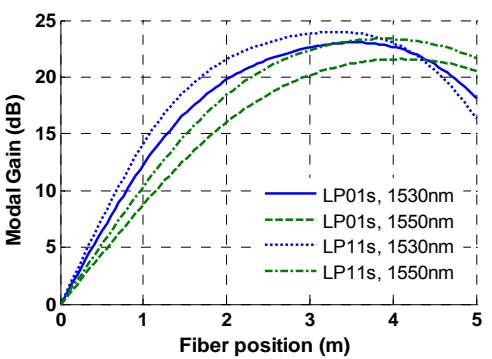

(d)

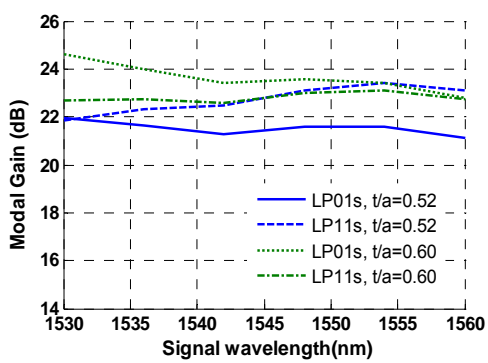

Fig. 2: (a) Fiber 2 with ringed doped profile (shaded region) and supported signal modes; a, core radius; $t$, thickness of the doped region. (b) Differential gain between $L_{01 \mathrm{~s}}$ and $L P_{11 \mathrm{~s}}$ (at 1530nm) versus input signal power for different t/a ratios. (c) Modal gain evolution of LP01s and LP11s along the fiber position for two different wavelengths $(1530 \mathrm{~nm}$ and $1550 \mathrm{~nm})$, using $\mathrm{t} / \mathrm{a}=0.52$. (d) Modal gain dependence on wavelength.

To illustrate the benefits to be derived from this design we plot the variation of DMG between $\mathrm{LP}_{01 \mathrm{~s}}$ and $\mathrm{LP}_{11 \mathrm{~s}}$ signal beams in a length of Fiber 2 forward pumped by a pure $L P_{01 p} 980 \mathrm{~nm}$ pump beam for various values of normalised ring thickness (defined by the ratio t/a as shown in Fig. 2a). The signal wavelength was chosen to be $1530 \mathrm{~nm}$ and pump power was fixed at $250 \mathrm{~mW}$. It should be noted that the pump absorbtion per unit length increases with increasing t/a. Thus for a fair comparision, the Fiber Length (FL) is adjusted to ensure the total output signal power (the sum of $L P_{01 s}$ and $L P_{11 s}$ ) reaches a maximum for each t/a value. The DMG values for a series of $t / a$ values and corresponding FL used are shown in Fig. 2b. As can be seen for values of $t / a$ around 0.53 very low levels of DMG can be obtained for a wide range of input powers. Moreover, through fine tuning of $t / a$, both negative and positive values of DMG can be achieved. For comparison, we have plotted the DMG for the conventional uniformly doped SI-EDF (with $t / a=1$ ), which remains large and positive at all input powers. In practice, we need to consider modal gain for the entire C-band. To investigate this aspect we selected signal wavelengths at $1530 \mathrm{~nm}$ and $1550 \mathrm{~nm}$ and chose a ratio for $\mathrm{t} / \mathrm{a}=0.52$ so as to have higher gain for the higher order modes. The $L P_{01 p}$ pump power was set at $250 \mathrm{~mW}$ whilst an input power of $-10 \mathrm{dBm}$ was used for both $L P_{01 s}$ and $L P_{11 s}$. The corresponding signal power evolution along the fiber is shown in Fig. 2c. At a fiber length of $\sim 3.5 \mathrm{~m}$, the gains for signals at $1530 \mathrm{~nm}$ are already saturated whilst those at $1550 \mathrm{~nm}$ are still increasing, which indicates that the fiber positions where the minimum absolute DMG value appears for signals at $1530 \mathrm{~nm}$ and $1550 \mathrm{~nm}$ are different. However, it is possible to minimize the gain excursion to within $1.5 \mathrm{~dB}$ across the full C-band for both $\mathrm{LP}_{01 \mathrm{~s}}$ and $\mathrm{LP}_{11 \mathrm{~s}}$ by choosing an EDF length of $4.3 \mathrm{~m}$, as depicted in Fig. 2d. Similarly, by adjusting the EDF length to $3.5 \mathrm{~m}$ with a ratio of $t / a=0.6$, we are able to obtain higher gain for the $\mathrm{LP}_{01 \mathrm{~s}}$ with minimal gain excursion $(\sim 2 \mathrm{~dB})$ across the C-band (as shown in Fig. 2d).

\section{Conclusions}

We report an experimentally validated tool for the modeling of FM-EDFAs. Using this tool we propose a novel ring-doped fiber design capable of providing accurate control of DMG in a TMEDFA for a significantly simplified (and much more practical) $\mathrm{LP}_{01}$-only based pumping configuration. We believe this ring-doped fiber design offers significant practical benefits relative to other TM-EDF designs proposed to date.

\section{Acknowledgements}

This work was supported in part by the EU 7th Framework Programme under grant agreement 228033 (MODE-GAP).

\section{References}

[1] R. Ryf et al., ECOC PDP Th.13.K.5. (2011)

[2] N. Bai et al., Opt. Express 20, 2668 (2012)

[3] Y. Jung et al., Opt. Express 19, B952 (2011)

[4] Z. Jiang et al., J. Opt. Soc. Am. B 25, 247 (2008)

[5] N. Bai et al., Opt. Express 19, 16601 (2011)

[6] W.L. Barnes et al., IEEE Journal of 27, 1004 (1991) 